\title{
Influence of the apical enlargement size on the endotoxin level reduction of dental root canals
}

\author{
Ariane Cassia Salustiano MARINHO ${ }^{1}$, Frederico Canato MARTINHO ${ }^{2}$, Alexandre Augusto ZAIA ${ }^{3}$, Caio Cezar Randi \\ FERRAZ3 ${ }^{3}$, Brenda Paula Figueiredo de Almeida GOMES ${ }^{4}$
}

\footnotetext{
1- BDS, Graduate student, Department of Restorative Dentistry, Endodontics Division, Piracicaba Dental School, State University of Campinas - UNICAMP, Piracicaba, SP, Brazil.

2- BDS, MSc, PhD, Research Professor, Department of Restorative Dentistry, Endodontics Division, Piracicaba Dental School, State University of Campinas - UNICAMP, Piracicaba, SP, Brazil.

3- BDS, MSc, PhD, Associate Professor, Department of Restorative Dentistry, Endodontics Division, Piracicaba Dental School, State University of Campinas - UNICAMP, Piracicaba, SP, Brazil.

4- DDS, MSc, PhD, Full Professor, Department of Restorative Dentistry, Endodontics Division, Piracicaba Dental School, State University of Campinas UNICAMP, Piracicaba, SP, Brazil.
}

Corresponding address: Dr. Brenda PFA Gomes - Faculdade de Odontologia de Piracicaba - UNICAMP - Departamento de Endodontia - Av. Limeira, 901 - Areião - Piracicaba - São Paulo - Brasil - 13414-903 - E-mail: bpgomes@fop.unicamp.br Phone: (55) 1921065343 - Fax: (55) 1921065218

Received: March 1, 2012 - Modification: August 16, 2012 - Accepted: September 17, 2012

\section{ABSTRACT}

\begin{abstract}
Gram-negative bacteria play an essential role in endodontic infections because they have virulence factors such as endotoxin. Due to its potential cytotoxic activity, special attention has been given to the removal/neutralization of this endotoxin in the root canal system. Objective: The aim of this study was to evaluate the influence of the apical enlargement size (AES) by using rotary instruments on the endotoxin level reduction of dental root canals. Material and Methods: Forty root canals of the mandibular premolar teeth were used. Escherichia coli endotoxin (055: B55) was inoculated into thirty root canals. Ten teeth served as the negative control group. After the incubation period, the first endotoxin samples were collected from the root canals with a sterile/apyrogenic paper point for the analysis of the endotoxin units (EU/mL) present before instrumentation (S1). Specimen instrumentation was performed with the Mtwo ${ }^{\circledR}$ rotary system in the sequence 10/.04, 15/.05, 20/.06, 25/.06, 30/.05, 35/.04 and 40/.04. To monitor the effectiveness of increasing apical enlargement on endotoxin removal, the second endotoxin samples were collected from all the root canals after instrumentation with the following instruments: \#25/.06- (S2); \#30/.05- (S3); \# 35/.04- (S4); and \#40/.04- (S5). Limulus amebocyte lysate (LAL) was used to quantify the levels of endotoxin. The results were statistically compared by using repeated measures of ANOVA with post hoc Tukey testing. Results: Increasing levels of endotoxin removal was achieved by large sized apical enlargement: S2 (AES \#25/.06)- 89.2\%, S3 (AES \#30/.05)- 95.9\%, S4 (AES \#35/.04)- 97.8\% and S5 (AES \#40/.04)- 98.2\%. Substantial reduction of endotoxin content was obtained in S4 and S5 compared to $S 2(p<0.05)$, however, the root canal preparation was not able to eliminate the endotoxin. Conclusions: Under the conditions of this study, it was concluded that the reduction of endotoxin levels of the dental root canals could be predicted by increasing the apical enlargement size.
\end{abstract}

Key words: Root canal. Bacterial infections. Instrumentation.

\section{INTRODUCTION}

Clinical investigations of endodontic infection have elucidated the strong correlation between oral bacterial lipopolysaccharide (LPS, known as endotoxin) present in the outer cell membranes of
Gram-negative bacteria ${ }^{12}$, and the presence of clinical symptomatology and apical periodontitis $s^{4,8,9,16,24}$.

Particularly in the periapical tissues, endotoxin can stimulate cells to release pro-inflammatory cytokines related to the development and maintenance of the inflammatory response, 
including bone resorption, even at very low concentrations ${ }^{8,10,14,16}$. Due to its potential cytotoxic activity in the pulp and periapical tissues, special attention has been given to the complete removal/ neutralization of endotoxin from infected root canals ${ }^{9,15}$

The clinical efficacy of endodontic procedures on bacterial LPS has been investigated in the endodontic literature $8,10,13,15-16$. Gomes, et al. ${ }^{7}$ (2009) had evaluated the action of chemo-mechanical preparation with hand files and $2.5 \% \mathrm{NaOCl}$ or $2 \%$ chlorhexidine $(\mathrm{CHX})$ gel as auxiliary chemical substances for the removal of endotoxin from the infected root canals. Despite the high antimicrobial activity of these auxiliary chemical substances, neither $2.5 \% \mathrm{NaOCl}$ nor $2 \% \mathrm{CHX}$ gel were effective in eliminating endotoxin. The authors suggested that the reduction of only $47 \%$ of the endotoxin content was related to the mechanical action of the instruments in the dentinal walls, accomplished by the flow and backflow of the irrigants. Martinho, et al. $^{15}$ (2010) indicated a reduction of $96.27 \%$ of the endotoxin contents by using rotary files accomplished by sterile/apyrogenic saline solution as an irrigant.

With regard to the optimal shape and apical size of the root canal preparation, there is some controversy on whether apical enlargement is required $1-2,8,14,18,23,28$. As a rule, the most important criteria for root canal enlargement should rely on the clinical determination of the initial apical file size $^{8}$. One recommended approach is to apically enlarge the root canal up to three sizes larger than the first file to bind.

The latest generation of root canal instruments includes nickel-titanium ( $\mathrm{Ni}-\mathrm{Ti}$ ) rotary systems. The super-elastic property of $\mathrm{Ni}$-Ti has allowed for a better centering ability of the instrument within the root canal lumen ${ }^{15}$. Besides, these instruments have reduced operator fatigue, procedural errors, and the duration of the canal preparation ${ }^{25}$. Mtwo ${ }^{\circledR}$ rotary files (VDW, Munich, Germany) stand out as a new generation of rotary instruments ${ }^{20}$. The files have two cutting edges with minimal radial contact providing maximum space for dentinal debris removal. The system is comprised of 7 tip sizes (10-40) with constant tapers ranging between 0.04 - 0.07. Thereby, final apical enlargement can be perfomed up to \#25/.0622, \#30/.055,22-24, \#35/.04 21,25 or \#40/.04 ${ }^{18,26,28}$.

In view of the widespread use of the rotary files, clinical investigations have evaluated the performance of Mtwo ${ }^{\circledR}$ rotary files for root canal instrumentation regarding cleaning and shaping ability, transportation of debris into the apex, preservation of the original geometry of the root canal, fracture risk and bacterial reduction ${ }^{21}$. However, no study evaluated the effectiveness of the increased apical enlargement by using rotary files for endotoxin reduction.

Therefore, the aim of this study was to evaluate the influence of apical enlargement size (AES) in reducing/eliminating endotoxin from root canals prepared with Mtwo ${ }^{\circledR}$ rotary files.

\section{MATERIAL AND METHODS}

\section{Specimen selection and preparation}

This study was submitted to and approved by the Ethics Committee of Research of the Piracicaba Dental School, State University of Campinas, UNICAMP (Protocol \#186/09). Forty canals of freshly extracted human mandibular pre-molars were used in the study. These teeth were collected from a general dental practice and had been extracted for pulpal or periodontal reasons. The teeth were stored in a $0.9 \%$ saline solution until use.

In order to be included in this study, all teeth had to present a mature root apex with no evidence of external resorption and a single narrow straight root canal. Preoperative radiographs were taken to ensure that the teeth did not have root caries, fractures, multiples canals, lateral radicular canals, calcifications or curvatures. Specimen selection was made on the basis of relative dimensions and similarity in root morphology. None of these teeth had received prior root canal treatment. Debris, calculus, and soft tissue remnants on the root surfaces were removed by using periodontal curettes (Golgran- Millennium, São Paulo, SP, Brazil). The crowns were transversely sectioned at the level of the cementum-enamel junction by using a water-cooled diamond disk (KGSorensen, Barueri, SP, Brazil). The root length was standardized to $15 \mathrm{~mm}$. Apical patency was determined by inserting a size $08 \mathrm{~K}$-file (DentsplyMaillefer, Ballaigues, Switzerland) into the root canal until the tip was visible at the apical foramen. The root canals were instrumented up to a \#20 K-file at the apical foramen in alternated rotation, under irrigation with $5 \mathrm{~mL}$ of distilled water after each file change. The smear layer was removed with $5.25 \% \mathrm{NaOCl}$ (Drogal, Piracicaba, SP, Brazil) for 10 minutes under constant agitation (AgitadorAquecedor Fanem, São Paulo, SP, Brazil), followed by $17 \%$ EDTA (Drogal, Piracicaba, SP, Brazil). The teeth were then washed thoroughly with distilled water for 1 hour $^{6}$, and then the canals were dried by using paper points (Dentsply, Rio de Janeiro, RJ, Brazil). The apical region was sealed with light-cured resin composites (3M Dental Products, St Paul, MN, USA) to prevent any permeability in the apical region. Next, the outer surfaces of the specimens were covered with two layers of epoxy resin (Araldite; Brascola, São Paulo, SP, Brazil), except for the cervical opening. 
All specimens were sterilized with autoclave (Fanem, São Paulo, SP, Brazil) at $121^{\circ} \mathrm{C}$ for 20 minutes in distilled water and then randomly divided into 24-well cell culture plates (Corning Costar, Cambridge, MA, USA). To make both handling and instrumentation easier, the teeth were vertically fixed at the cervical region with chemically activated acrylic resin (Artigos Odontológicos Clássico, São Paulo, SP, Brazil) inside the wells. For the degradation of preexisting endotoxins, all materials used in the experiment were sterilized by gamut radiation with cobalt 60 (20 KGy for 6 hours) ${ }^{3}$.

\section{Specimen contaminated with endotoxin}

Before starting the experiment, a pilot study was made in order to ensure the endotoxin contamination protocol by the Limulus amebocyte lysate assay (LAL). After this, 30 teeth were inoculated with $30 \mu \mathrm{L}$ of a solution containing Escherichia coli 055: B55 endotoxin (1.000 EU/mL) and incubated for 24 hours at $37^{\circ} \mathrm{C}$ in a humidified atmosphere, according to Oliveira, et al. ${ }^{20}$ (2005). As a negative control group, ten teeth were inoculated with $30 \mu \mathrm{L}$ of LAL water (endotoxin-free) and incubated for 24 hours at $37^{\circ} \mathrm{C}$ in a humidified atmosphere.

\section{Sampling and instrumentation procedure}

After the incubation period, all the root canals were sampled to determine the initial levels of endotoxin present before chemo-mechanical preparation (S1). A sterile/apyrogenic absorbent paper point was introduced into the full extension of the root canal and remained in position for 60 seconds. It was then placed in an endotoxin-free glass and frozen at $-20^{\circ} \mathrm{C}$ for further quantification of the endotoxin. Mtwo ${ }^{\circledR}$ was carried out according to the manufacturer's instructions (sequence 10/.04, $15 / .05,20 / .06,25 / .06,30 / .05,40 / .04$ ) into the full extension of the root canal with a gentle in-and-out motion. Once the instrument had achieved the end of the canal and rotated freely, it was removed ${ }^{25}$. The working time of each instrument was monitored. In order to avoid possible cutting blade interference, the $\mathrm{Mtwo}^{\circledR}$ files were used once. The canals were shaped by using $1 \mathrm{~mL}$ of non-pyrogenic water that was renewed with every change of instrument. All the experimental procedures were conducted by the same operator. To evaluate the effectiveness of increased apical enlargement on the endotoxin levels, the root canals were sampled with a sterile/ apyrogenic absorbent paper point, after using the following instruments: \#25/.06- as S2 (basic series); \#30/.05- as S3; \#35/.04- as S4; and \#40/.04- as S5 (shown in Table 1).

\section{Determination of endotoxin concentration \\ The turbidimetric test (Pyrogent 5000 ${ }^{\circledR}$, BioWhitaker, Cambrex Co, Walkersville, MD, USA)} was used to measure the endotoxin levels in the root canals via the $\mathrm{LAL}$ technique, previously published by the authors ${ }^{13,15-16}$. It is a kinetic, quantitative assay for the detection of endotoxin, in which the activated LAL converts the coagulogen into coagulin to produce turbidity in the sample. All the samples were mixed with the reconstituted LAL reagent, placed in a photometer, and automatically monitored over time until the appearance of turbidity. The time required before the appearance of turbidity (reaction time) was inversely proportional to the amount of endotoxin. In the presence of a large amount of endotoxin, the reaction occurred rapidly, in the presence of a small amount of endotoxin, the reaction time was increased. The concentration of endotoxin within the unknown samples was calculated from a standard curve. Its wide-range sensitivity [0.01-100 endotoxin units $(E U / m L)]$ requires small amounts of dilutions of the clinical samples, thus optimizing the laboratory time. As a parameter for the calculation of the amount of endotoxins existing in the root canal samples, a standard curve was plotted by using the endotoxin of a known concentration supplied by the kit (100 EU/mL), with its dilutions reaching

Table 1- Evaluation of increasing apical enlargement of $\mathrm{Mtwo}^{\circledR}$ rotary files on endotoxin levels

\begin{tabular}{cccc}
\hline $\begin{array}{c}\text { APICAL ENLARGEMENT } \\
\text { SIZE (S2) }\end{array}$ & $\begin{array}{c}\text { APICAL ENLARGEMENT } \\
\text { SIZE (S3) }\end{array}$ & $\begin{array}{c}\text { APICAL ENLARGEMENT } \\
\text { SIZE (S4) }\end{array}$ & $\begin{array}{c}\text { APICAL ENLARGEMENT } \\
\text { SIZE (S5) }\end{array}$ \\
\hline $10 / .04$ & $10 / .04$ & $10 / .04$ & $10 / .04$ \\
$15 / .05$ & $15 / .05$ & $15 / .05$ & $15 / .05$ \\
$20 / .06$ & $20 / .06$ & $20 / .06$ & $20 / .06$ \\
$25 / .06$ & $25 / .06$ & $25 / .06$ & $25 / .06$ \\
& $\mathbf{3 0 / . 0 5}$ & $30 / .05$ & $30 / .05$ \\
SAMPLING PROCEDURE & & $\mathbf{3 5 / . 0 4}$ & $35 / .04$ \\
& SAMPLING PROCEDURE & SAMPLING PROCEDURE & $\mathbf{4 0 / . 0 4}$ \\
& & & SAMPLING PROCEDURE \\
\hline
\end{tabular}

Bold font highlights the final apical instruments in each group 
Table 2- Mean and standard deviation $( \pm S D$ ) values of the levels of endotoxin reduced by different apical preparations size tested of $\mathrm{Mtwo}^{\circledR}$ rotary files

\begin{tabular}{|c|c|c|}
\hline Apical enlargement size tested & Levels of endotoxin (EU/mL) & Percent reduction \\
\hline & \multicolumn{2}{|c|}{ Mean value $\pm S D$} \\
\hline S1 (before instrumentation) & \multicolumn{2}{|c|}{$630( \pm 10.69)$} \\
\hline S2 (AES \#25/.06) & $68.0( \pm 20.76)$ & $89.2( \pm 15.76)^{a}$ \\
\hline S3 (AES \#30/.05) & $25.3( \pm 25.65)$ & $95.9( \pm 16.25)^{\mathrm{ab}}$ \\
\hline S4 (AES \#35/.04) & $13.6( \pm 15.69)$ & $97.8( \pm 12.86)^{\mathrm{bc}}$ \\
\hline S5 (AES \#40/.04) & $10.8( \pm 12.31)$ & $98.2( \pm 10.49)^{c}$ \\
\hline
\end{tabular}

Mean values followed by different letters indicate statistical significant difference among the sequences tested $(p<0.05)$

the final concentrations $(0.01,0.1,1,10 \mathrm{EU} / \mathrm{mL})$ according to the manufacturer's instructions. A 96-well microplate (Corning Costar, Cambridge, MA, USA) was used in a heating block at $37^{\circ} \mathrm{C}$ and maintained at this temperature throughout the assay. First, the endotoxin samples were suspended in $1 \mathrm{~mL}$ of LAL water supplied by the kit and agitated in vortex for 60 seconds. Immediately after, 100 $\mathrm{mL}$ of the blank, followed by the same volume of the standard endotoxin solutions $(0.01,0.1,1,10$ $\mathrm{EU} / \mathrm{mL}$ ) and $100 \mathrm{~mL}$ of the samples, were added in duplicate to the 96-well microplate with their respectively positive controls. To avoid inhibition or enhancement of the $L A L$, a known concentration of Escherichia coli endotoxin was added to the clinical samples, as recommended by the manufacturer's instructions (spike procedure). Both the unspiked and spiked samples (control) had their endotoxin concentrations automatically calculated. The endotoxins recovered were equal to the known spike concentration ranging from $50 \%$ to $200 \%$. All reactions were performed in duplicate to validate the test. The test procedure was performed according to the manufacturer's instructions. The absorbance of endotoxins was individually measured by using an enzyme-linked immunosorbent assay plate reader (Ultramark, Bio-Rad Laboratories Inc, Hercules, CA, USA). During the calculation of the endotoxin concentration, the microplate reader, by using the WinKQCL Software (BioWittaker, Cambrex Co, Walkersville, MD, USA), monitors the absorbance of each well of the microplate continuously at $340 \mathrm{~nm}$ throughout the assay. By using the initial absorbance reading of each well as its own blank, the reader determines the time required for absorbance at 0.03 absorbance units. This time is termed as the reaction time. The WinKQCL Software automatically performs a log/log linear correlation of the reaction time of each standard curve with its corresponding endotoxin concentration, including printing the standard curve parameters. If the absolute value of the correlation coefficient $(r)$ is 0.980 , a polynomial model can be used to construct a standard curve and in turn predict the endotoxin concentrations of the test samples.

\section{Statistical analysis}

The data obtained with the LAL assay was statistically analyzed by using the SAS Software, version 9.1 (SAS Institute, Cary, NC, USA). The Shapiro-Wilk test was used to verify the normal data distribution. Both the mean and standard deviation regarding the endotoxin levels after each sampling procedure were determined. A one way repeated measures ANOVA was applied. When significant differences were found between the groups, the Tukey's test was subsequently used. The significance levels were always set at $5 \%$ $(p<0.05)$.

\section{RESULTS}

The Shapiro-Wilk test showed data normality ( $p>0.266)$. For the validation of the LAL assay, the standard curve fulfilled the criteria of linearity for all running assays, as reported by the guidelines. The LAL assay indicated that endotoxins were present in $100 \%$ of the root canals investigated $(30 / 30)$ with a median value of $630 \mathrm{EU} / \mathrm{mL}$. Sterility of the specimens was confirmed with the negative control group showing an absence of endotoxin in all the root canals (10/10). As a result of the chemomechanical preparation, the LPS content was reduced to a mean value of $10.8 \mathrm{EU} / \mathrm{mL}$. The mean values and the standard deviation of the different levels of endotoxin reduction are shown in Table 2.

\section{DISCUSSION}

The present study evaluated the influence of the apical enlargement size (AES) in reducing/ eliminating endotoxin in the root canals prepared with Mtwo ${ }^{\circledR}$ rotary files. Analyses of the results indicated that a root canal preparation larger than a \#30/.05 file showed the best performance regarding endotoxin removal. Additionally, the size 
of the apical enlargement implicated significantly in the removal of endotoxins from the root canals.

At the base line samples, after 24 hours of incubation time, endotoxins were recovered from $100 \%$ of the root canals sampled, validating the contamination protocol in the present study. Nissan, et al. ${ }^{19}$ (1995) evaluated the diffusion capacity of bacterial endotoxin through human dentin in the apical direction and concluded that endotoxin was capable of passing through $0.5 \mathrm{~mm}$ of dentin in a period that ranged from $15 \mathrm{~min}$ to $4 \frac{1}{2} \mathrm{~h}$, suggesting that the dentin may allow the passage of bacterial products to the pulpal space. Oliveira, et al.20 (2005) showed that endotoxin can diffuse through the dentinal tubules towards the cement in $24 \mathrm{~h}$.

In clinical practice, the most frequently Mtwo ${ }^{\circledR}$ sequence used, the basic series, ending in an apical enlargement size of \#25/.06, was able to reduce endotoxin contents by $89.2 \%$ (S2). In fact, considering the apical diameter of the lower premolars, which corresponds to that of a \#25 or a \#30 file ${ }^{27}$, it was expected that the enlargement to a \#25 file-size would not significantly improve the disinfection of the root canal system, as demonstrated by the present study.

Clinically, as a rule, the root canal enlargement relies on the determination of the initial apical file size $^{8}$. Then the canal should be apically enlarged up to three sizes larger than the first file to bind the working length. In order to achieve a more accurate measurement of the initial apical diameter, the removal of interferences by cervical pre-flaring should be considered previously ${ }^{8,18}$.

Our data indicated that the root canal enlargement achieved by a final apical file larger than \#30/.05, showed the best performance in removing endotoxin from the dentinal walls. In agreement, Martinho, et al. ${ }^{15}$ (2010) evaluating the ability of the root canal procedures on eliminating endotoxins, demonstrated a $96.27 \%$ reduction of endotoxin, achieved by a root canal enlargement up to a \#30 file size. In fact, an apical enlargement wider than ISO \#30 is recommended to remove not only endotoxin (as shown in the present work), but also the more infected dentin. Also, this approach allows deeper irrigation and contributes to enclosing the canal cross-sections, accessory canals and apical deltas $5,8,11,17,23$.

Regardless of the apical enlargement size, endotoxins were still detected in $100 \%$ of the root canals after instrumentation. Whether the residual amount of endotoxins is able to induce a periapical inflammatory response is not known. Theoretically, in the absence of living Gram-negative cells to maintain the LPS levels in the periapical tissues, the effect of these residual levels of endotoxins left behind after the root canal instrumentation, would only temporarily modulate the host defenses, without impairing the endodontic treatment outcome.

Overall, our study demonstrated that a root canal preparation, with a file larger than \#30/.05, achieves a better removal of endotoxins from the root canals. Moreover, the determination of the initial apical file size is one of the most important steps to determine a correct apical enlargement diameter to be achieved during root canal instrumentation, particularly, when approaching a root canal enlargement based on the "three sizes up from the first file to bind" rule.

\section{CONCLUSION}

Based on the results of this research, endotoxin levels of dental root canals could be predicted by increasing the apical enlargement size. Root canal preparation was effective in reducing the amount of endotoxin within the infected root canals. Further studies should be performed with different rotary systems associated with irrigation devices to establish protocols for a more effective root canal cleaning.

\section{ACKNOWLEDGMENTS}

We would like to thank Ana Regina de Oliveira Polay for technical support. We are also thankful to Lonza for the Kinetic-QCL equipment. This work was supported by the Brazilian agencies FAPESP (10/51113-1; 10/17877-4; 10/19136-1; 11/50510$0)$ and CNPq (302575/2009-0; 150557/2011-6). The authors deny any conflicts of interest.

\section{REFERENCES}

1- Baugh D, Wallace J. The role of apical instrumentation in root canal treatment: a review of the literature. J Endod. 2005;31:33340.

2- Coldero LG, McHugh S, MacKenzie D, Saunders WP. Reduction in intra-canal bacteria during root canal preparation with and without apical enlargement. Int Endod J. 2002;35:437-46.

3- Csako G, Elin RJ, Hochstein HD, Tsai CM. Physical and biological properties of U.S. standard endotoxin EC after exposure to ionizing radiation. Infect Immun. 1983;41:190-6.

4- Dahlén G, Bergenholtz G. Endotoxic activity in teeth with necrotic pulps. J Dent Res. 1980;59:1033-40.

5- Falk KW, Sedgley CM. The influence of preparation size on the mechanical efficacy of root canal irrigation in vitro. J Endod. 2005;31:742-5.

6- Ferraz CC, Gomes BP, Zaia AA, Teixeira FB, Souza-Filho FJ. In vitro assessment of the antimicrobial action and mechanical ability of chlorhexidine gel as an endodontic irrigant. J Endod. 2001;27:452-5.

7- Gomes BP, Martinho FC, Vianna ME. Comparison of $2.5 \%$ sodium hypochlorite and $2 \%$ chlorhexidine gel on oral bacterial lipopolysaccharide reduction from primarily infected root canals. J Endod. 2009;35:1350-3.

8- Hecker H, Bartha T, Löst C, Weiger R. Determining the apical preparation size in premolars: part III. Oral Surg Oral Med Oral Pathol Oral Radiol Endod. 2010;110:118-24. 
9- Horiba N, Maekawa Y, Abe Y, Ito M, Matsumoto T, Nakamura $\mathrm{H}$. Correlations between endotoxin and clinical symptoms or radiolucent areas in infected root canals. Oral Surg Oral Med Oral Pathol. 1991;71:492-5.

10- Horiba N, Maekawa Y, Matsumoto T, Nakamura H. A study of the distribution of endotoxin in the dentinal walls of infected root canals. J Endod. 1990;16:331-4.

11- Khademi A, Yazdizadeh M, Feizianfard M. Determination of the minimum instrumentation size for penetration of irrigants to the apical third of root canal systems. J Endod. 2006;32:417-20. 12- Lüderitz O, Tanamoto K, Galanos C, McKenzie GR, Brade H, Zähringer $U$, et al. Lipopolysaccharides: structural principles and biologic activities. Rev Infect Dis 1984;4:428-3.

13- Martinho FC, Gomes BP. Quantification of endotoxins and cultivable bacteria in root canal infection before and after chemomechanical preparation with $2.5 \%$ sodium hypochlorite. J Endod. 2008;34:268-72.

14- Martinho FC, Chiesa WM, Leite FR, Cirelli JA, Gomes BP. Antigenic activity of bacterial endodontic contents from primary root canal infection with periapical lesions against macrophage in the release of interleukin-1beta and tumor necrosis factor alpha. J Endod. 2010;36:1467-74.

15- Martinho FC, Chiesa WM, Marinho AC, Zaia AA, Ferraz CC, Almeida JF, et al. Clinical investigation of the efficacy of chemomechanical preparation with rotary nickel-titanium files for removal of endotoxin from primarily infected root canals. J Endod. 2010;36:1766-9.

16- Martinho FC, Chiesa WM, Zaia AA, Ferraz CC, Almeida JF, Souza-Filho FJ, et al. Comparison of endotoxin levels in previous studies on primary endodontic infections. J Endod. 2011;37:163-7. 17- McGurkin-Smith R, Trope M, Caplan D, Sigurdsson A. Reduction of intra-canal bacteria using GT rotary instrumentation, 5.25\% $\mathrm{NaOCl}$, EDTA, and $\mathrm{Ca}(\mathrm{OH}) 2$. J Endod. 2005;31:359-63.
18- Mickel AK, Chogle S, Liddle J, Huffaker K, Jones JJ. The role of apical size determination and enlargement in the reduction of intra-canal bacteria. J Endod. 2007;33:21-3.

19- Nissan R, Segal H, Pashley D, Stevens R, Trowbridge H. Ability of bacterial endotoxin to diffuse through human dentin. J Endod. $1995 ; 21: 62-4$.

20- Oliveira LD, Carvalho CA, Valera MC, Koga-Ito CY, Jorge AO. Diffusion ability of endotoxin through dentinal tubules. Braz Oral Res. 2005;19:5-10.

21- Paqué F, Ganahl D, Peters OA. Effects of root canal preparation on apical geometry assessed by micro-computed tomography. J Endod. 2009;35:1056-9.

22- Parris J, Wilcox L, Walton R. Effectiveness of apical clearing: histological and radiographical evaluation. J Endod. 1994;20:21924.

23- Pitts DL, Williams BL, Morton TH Jr. Investigation of the role of endotoxin in periapical inflammation. J Endod. 1982;8:10-8.

24- Schein B, Schilder H. Endotoxin content in endodontically involved teeth. 1975. J Endod. 2006;32:293-5.

25- Sonntag D, Ott M, Kook K, Stachniss V. Root canal preparation with the NiTi systems K3, Mtwo and ProTaper. Aust Endod J. 2007;33:73-81.

26- Usman N, Baumgartner JC, Marshall JG. Influence of instrument size on root canal debridement. J Endod. 2004;30:110-2.

27- Wu MK, Barkis D, Roris A, Wesselink PR. Does the first file to bind correspond to the diameter of the canal in the apical region? Int Endod J. 2002;35:264-7.

28- Yared GM, Bou Dagher FE. Apical enlargement: influence on overextensions during in vitro vertical compaction. J Endod. 1994;20:269-71. 